\title{
AUTHOR INDEX, VOLUME 1 (1969) - VOLUME 10 (1974)
}

\section{H. Adams}

The completion by cuts of an orthocomplemented modular lattice

The completion by cuts of an orthocomplemented modular lattice: Addendum

Baer semigroups and lattices (A)*

Helen E. Adams

Filtrations and valuations on rings (A)

John K. Adams

The theory of waves in dispersive systems and its application to problems in physical oceanography (A)

P. W. Aitchison

Some characterisations of the ellipsoid and the Minkowski theory of reduction (A)

Michael D. Alder

Remark on a theorem of E. H. Brown

\section{R. R. Allan}

Delay-flow relationships in continuously distributed traffic (A)

G. D. Allen, C. K. Chui, W. R. Madych, F. J. Narcowich, and P. W. Smith

Padé approximation and orthogonal polynomials

Brian R. Alspach and Norman J. Pullman

Path decompositions of digraphs

Ronald Alter and Mark Villarino

A remark on primes in arithmetic progressions

Mieczyslaw Altman

A class of majorant functions for contractors and equations

A. R. Amir-Moéz and C. R. Perry

Remarks on theorems of Thompson and Freede

L. W. Anderson

See R. P. Hunter

R. S. Anderssen

A class of densely invertible parabolic operator equations

Exact variational representations for the solution of the simple parabolic equation

\section{A. L. Andrew}

Convergence of approximate operator methods for eigenvectors

Alan L. Andrew

Variational solution of certain nonlinear eigenvalue problems (A)

\section{A. L. Andrew and G. C. Elton}

Computation of eigenvectors corresponding to multiple eigenvalues

Howard Anton

On Lebesgue-type decompositions for Banach algebras

Howard Anton and W. J. Pervin

A Hahn-Banach theorem for complex semifields

* (A) denotes an abstract of a PhD Thesis. $\begin{array}{rrr}1 & (1969) & 279-280 \\ 1 & (1969) & 432 \\ 3 & (1970) & 425-426 \\ & & \\ 5 & (1971) & 425-426\end{array}$

7 (1972) 305-306

$2(1970) \quad 280$

9 (1973) $55-60$

2 (1970) 135-136

$10 \quad(1974) \quad 263-270$

10 (1974) 421-427

3 (1970) 185-192

$10 \quad(1974) \quad 51-58$

5 (1971) 221-225

$1 \quad(1969) \quad 363-374$

5 (1971) 305-314

3 (1970) 199-205

5 (1971) 137-138

4 (1971) 419-422

3 (1970) 39- 47

2 (1970) 129-133 


\section{James Demetrios Argyros}

A study of the redistribution of line radiation scattered in a plasma (A)

$4 \quad(1971) \quad 425-426$

\section{Ludwig Arnold}

The $\log \log$ law of multidimensional stochastic integrals and diffusion processes

5 (1971) $351-356$

\section{Shashi Prabha Arya}

See M. K. Singal

S. Asghar and M. A. Rashid

Diffraction of planetary waves by an infinite strip

T. W. Atterton

A note on certain subsets of algebraic integers

A prime ideal and its quotient ring

C. E. Aull

A generalization of a theorem of Aquaro

Christine Ayoub

See Raymond G. Ayoub

Raymond G. Ayoub and Christine Ayoub

On the group ring of a finite abelian group

1 (1969) 245-261

\section{G. Bachman}

See L. Narici

\section{S. Bachmuth and $\mathrm{H}$. Y. Mochizuki}

Third Engel groups and the Macdonald-Neumann conjecture

Kalyan Kumar Bagchi and Lokenath Debnath

Transient development of capillary-gravity waves in a running stream

Ivan Baggs

Maximal perfect spaces

10 (1974) 293-304

1 (1969) 345-352

3 (1970) $107-110$

$9 \quad(1973) \quad 105-108$

Beverley June Balkau

Topics in population genetics (A)

C. M. Bang

Partially well-ordered sets of infinite matrices and closed classes of abelian groups

D. W. Barnes

On complemented chief factors of finite soluble groups

Noel Geoffrey Barton

Interval layers in rotating fluids (A)

Gilbert Baumslag

Finitely generated cyclic extensions of free groups are residually finite

A finitely generated, infinitely related group with trivial multiplicator

A non-cyclic, locally free, free-by-cyclic group all of whose finite factor groups are cyclic

$\begin{array}{lrr}5 & (1971) & 379-386 \\ 9 & (1973) & 417-432 \\ 7 & (1972) & 429-436 \\ 9 & (1973) & 311\end{array}$

$10 \quad(1974) \quad 161-167$

7 (1972) $\quad 101-104$

$10 \quad(1974) \quad 147-148$

$5 \quad(1971) \quad 87-94$

5 (1971) 131-136

John A. Beachy

Cotorsion radicals and projective modules

6 (1972) 313-314

$5 \quad(1971) \quad 241-253$

E. Beckenstein

See L. Narici

Mehdi Behzad

The connectivity of total graphs

1 (1969) $175-181$

Howard E. Bell

Near-rings in which each element is a power of itself

$2 \quad(1970) \quad 363-368$

John Alan Belward

Forced convection and some related integral equations (A)

$6 \quad(1972) \quad 471-472$ 


\section{J. A. Belward}

Elastic waves in a prestressed Mooney material

Some dynamic properties of a prestressed incompressible hyperelastic material

\section{J. L. Berggren}

An algebraic characterization of finite symmetric tournaments

An algebraic characterization of symmetric graphs with a prime number of vertices

$$
\begin{array}{lll}
7 & (1972) & 135-160 \\
8 & (1973) & 61-73 \\
6 & (1972) & 53-59 \\
7 & (1972) & 131-134
\end{array}
$$

Leon Bernstein

See A. G. Shannon

L. Bican, P. Jambor, T. Kepka, P. Němec

On rings with trivial torsion parts

9 (1973) 275-290

\section{J. R. Blake}

Self propulsion due to oscillations on the surface of a cylinder at low Reynolds number

$\begin{array}{rrr}5 & (1971) & 255-264 \\ 5 & (1971) & 331-335 \\ & & \\ 9 & (1973) & 291-298 \\ 10 & (1974) & 59-66\end{array}$

Roger D. Bleier

Minimal vector lattice covers

Walter R. Bloom

A converse of Bernstein's inequality for locally compact groups

Jackson's Theorem for locally compact abelian groups

Nguyen-Huu-Bong

Some combinatorial and spectral properties of Baxter operators and related linear transformations (A)

\section{R. S. Booth}

Sequences defined as minima of two Fibonacci-type relations

\section{J. T. Borrego}

Adjunction semigroups

Ann K. Boyle

When projective covers and injective hulls are isomorphic

Alan Brace and D. E. Daykin

A finite set covering theorem

A finite set covering theorem II

A finite set covering theorem III

R. D. Braddock

Optimal problems in physical oceanography (A)

See also P. van den Driessche

\section{J. M. Brady}

On the classification of just-non-Cross varieties of groups

On soluble just-non-Cross varieties of groups

Just-non-Cross varieties of groups (A)

$4 \quad(1971) \quad 285-286$

$6 \quad(1972) \quad 75-81$

$1 \quad(1969) \quad 47-58$

$8 \quad(1973) \quad 471-476$

5 (1971) $\quad 197-202$

6 (1972) $19-24$

$6 \quad(1972) \quad 417-433$

2 (1970) $140-141$

J. M. Brady, R. A. Bryce and John Cossey

On certain abelian-by-nilpotent varieties

Warren Brisley

Some problems in varieties of groups (A)

Warren Brisley and L. G. Kovács

On soluble groups of prime-power exponent

Peter I. Brooker

Problem in kinetic theory (A)

M. S. Brooks, L. G. Kovács and M. F. Newman

A finite basis theorem for product varieties of groups

K. A. Broughan

Metrization of spaces having Čech dimension zero $\begin{array}{lll}3 & (1970) & 293-311 \\ 3 & (1970) & 313-323 \\ 3 & (1970) & 427-428 \\ 1 & (1969) & 403-416 \\ 2 & (1970) & 281-282 \\ 4 & (1971) & 389-396 \\ 3 & (1970) & 423-424 \\ 2 & (1970) & 39-44 \\ 9 & (1973) & 161-168\end{array}$ 


\section{A. Brown}

On the differential equations for tide-well systems

Proof of a conformal mapping relationship

A. Brown and E. N. Dancer

Functions of asymptotic expansions

Peter Joseph Brown

Long-period oscillations in rotating oceans (A)

Patrick J. Browne

Self adjoint operators and matrix measures

A class of linear operators (A)

Richard A. Brualdi

Comments on bases in dependence structures

A. M. Brunner

Fibonacci sequences and group theory (A)

R. M. Bryant, R. A. Bryce and B. Hartley

The formation generated by a finite group

R. M. Bryant, and L. G. Kovács

A note on generalized characters

The skeleton of a variety of groups

Nita Bryce

On the Mathieu group $M_{23}$ (A)

R. A. Bryce

Metabelian groups and varieties

Projective groups in varieties

See also J. M. Brady

See also R. M. Bryant

R. A. Bryce and John Cossey

Some product varieties of groups

Maximal Fitting classes of finite soluble groups

R. Burman

Particle paths of general relativity as geodesics of an affine connection

\begin{tabular}{rrr}
5 & $(1971)$ & $175-185$ \\
10 & $(1974)$ & $91-94$ \\
4 & $(1971)$ & $255-258$ \\
& & \\
6 & $(1972)$ & $476-477$ \\
4 & $(1971)$ & $289-305$ \\
4 & $(1971)$ & $429-430$ \\
1 & $(1969)$ & $161-167$ \\
9 & $(1973)$ & $473-474$ \\
& & \\
2 & $(1970)$ & $347-357$ \\
& & \\
5 & $(1971)$ & $265-269$ \\
6 & $(1972)$ & $357-378$ \\
3 & $(1970)$ & 139 \\
\hline 1 & $(1969)$ & $15-25$ \\
6 & $(1972)$ & $169-175$
\end{tabular}

R. R. Burman

Paths of charges in general relativity as geodesics of Einstein's non-Riemannian geometry

Geodesics of an affine connection and electromagnetism with radiation reaction

Lynette M. Butler

Certain non-algebras in harmonic analysis

R. N. Buttsworth

A family of groups with a countable infinity of full orders

C. C. Cadogan

On multigraphs with a given partition

Pauline Cain

Decomposition of complete graphs into stars

G. Calugareanu jr

See H. Wiesler

Alan R. Camina and F. Peter Lockett

On soluble groups which admit the dihedral group of order eight fixedpoint-freely

J. M. Campbell

A note on flasque sheaves

Torsion theories and coherent rings

$\begin{array}{rrr}3 & (1970) & 231-264 \\ 10 & (1974) & 169-175 \\ 3 & (1970) & 325-335\end{array}$

$4 \quad(1971) \quad 69-84$

$4 \quad(1971) \quad 225-240$

$4 \quad(1971) \quad 247-254$

4 (1971) 97-104

$3 \quad(1970) \quad 125-137$

$10 \quad(1974) \quad 23-30$ 


\section{K. Capell}

Asymptotic analysis of a linearized trailing edge flow

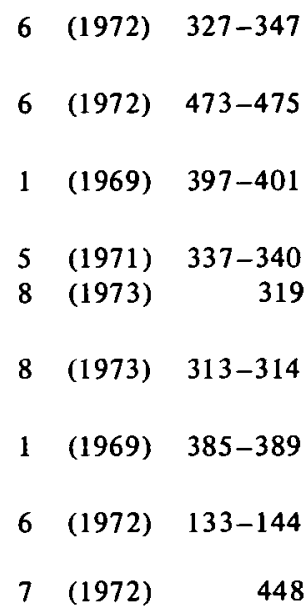

Kenneth Capell

Steady two-dimensional viscous flow in a jet and in a wake (A)

S. R. Caradus

The approximation problem for compact operators

Jon F. Carlson

Block idempotents and the Brauer correspondence

Block idempotents and the Brauer correspondence: Corrigendum

Frank A. Cezus

Green's relations in semigroups of functions (A)

M. Chacron

On algebraic rings

J. J. M. Chadwick

Schauder decompositions in non-separable Banach spaces

Bases of projections in Banach spaces with an appendix on non-standard analysis (A)

$7 \quad(1972)$

448

J. J. M. Chadwick and R. W. Cross

A characterization of pre-near-standardness in locally convex linear topological spaces

6 (1972) $107-115$

P. Chadwick

Interchange of modal properties in the propagation of harmonic waves in heat-conducting materials

Prem Chandra

A theorem on absolute summability of Fourier series by Riesz means

John W. Chaney

A note on the lattice properties of the linear maps of finite rank

T. C. Chau

The laws of some nilpotent groups of small rank (A)

Su-shing Chen

A dimension formula relating to algebraic groups

Chin-Hung Ching

Uniqueness and representation of a function in terms of its translated averages

$8 \quad(1973) \quad 75-92$

$3 \quad(1970) \quad 179-184$

$8 \quad(1973) \quad 343-353$

2 (1970) 277-279

$4 \quad(1971) \quad 241-245$

Chin-Hung Ching and Charles K. Chui

Representation of a function in terms of its mean boundary values

Uniqueness and nonuniqueness in mean boundary value problems

Some inequalities in trigonometric approximation

E. Chislett and C. S. Hoo

Coretraction-fibrations are retractions

C. Christensen

Rings with a few more zero-divisors

9 (1973) 121-125

$\begin{array}{lll}7 & (1972) \quad 425-427\end{array}$

8 (1973) 23- 26

8 (1973) $393-395$

$5 \quad(1971) \quad 363-374$

$5 \quad(1971) \quad 271-274$

C. K. Chui

See G. D. Allen

Chartes K. Chui

See Chin-Hung Ching

Philip T. Church and Klaus Lamotke

Almost free actions on manifolds

$10 \quad(1974) \quad 177-196$

B. E. Clancy

Multigroup neutron transport theory in plane geometry (A)

$5 \quad(1971) \quad 287-288$

John Clark

On the associativity of the torsion functor 


\section{J. Carke}

Oscillations within and on a fluid enclosed by arbitrary boundaries (A)

S. B. Conlon

An extension of the Krull-Schmidt theorem

S. B. Conlon and W. D. Wallis

The identity of certain representation algebra decompositions

\section{Howard John Connell}

Theory and applications of the interaction between surface waves and currents in water of varying depth (A)

\section{Paul F. Conrad}

Minimal vector lat tice covers

Countable vector lattices

Malcolm William Cook

Some results for relativistic space-times with spherical symmetry (A)

C. D. H. Cooper

Chunikhin's existence theorem for subgroups of a finite group

Joan Cooper

A note on Hadamard arrays

Joan Cooper and Jennifer Wallis

A construction for Hadamard arrays

W. A. Coppel

Matrix quadratic equations

W. A. Coppel and K. J. Palmer

Averaging and integral manifolds

William H. Cornish

Abelian Rickart semirings (A)

Congruences on distributive pseudocomplemented lattices

John Cossey and Sheila Oates Macdonald

On the definition of saturated formations of groups

John Cossey

See also J. M. Brady

See also R. A. Bryce

J. Graham Couper

Some analytical critical points in 3-space (A)

Michael G. Cowling

Extension of multipliers by periodicity

John H. Cozzens

Twisted group rings and a problem of Faith

B. D. Craven

A generalization of Lagrange multipliers

Two properties of Bochner integrals

B. D. Craven and B. Mond

A Fritz John theorem in complex space

R. W. Cross

See J. J. M. Chadwick

\section{R. S. Dahiya}

Nonoscillation of arbitrary order retarded differential equations of non-homogeneous type

See also Bhagat Singh

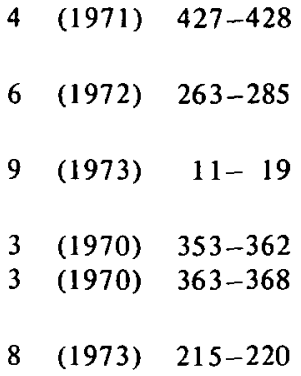

$5 \quad(1971) \quad 423-424$

8 (1973) $161-179$

4 (1971) 9- 15

7 (1972) $\quad 315-316$

$4 \quad$ (1971) $35-39$

$10 \quad(1974) \quad 371-376$

$10 \quad(1974) \quad 149-151$

$4 \quad(1971) \quad 397-406$

$10 \quad(1974) \quad 15-21$

$7 \quad(1972) \quad 269-277$

$10 \quad(1974) \quad 377-401$

$2(1970) \quad 197-222$

\section{5}

$4 \quad(1971) \quad 423-424$

1 (1969) 109-114

3 (1970) $73-74$ 


\section{E. N. Dancer}

See A. Brown

Susan Dancs

The sub-near-field structure of finite near-fields

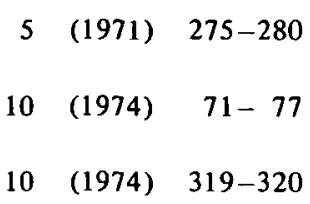

D. K. Datta

Recurrent tensors and holonomy group

3 (1970) $385-390$

A note on order topologies on ordered tensor products

Rings with orthogonality relations

4 (1971) 163-178

J. L. Davy

Properties of the solution set of a generalized differential equation

$\begin{array}{lll}6 & (1972) \quad 379-398\end{array}$

Brian Day

Construction of bi-closed categories (A)

Note on monoidal localisation

5 (1971) 139-140

8 (1973) $1-16$

D. E. Daykin

See Alan Brace

Lokenath Debnath

See Kalyan Kumar Bagchi

Madhukar G. Deshpande

A note on subdirectly irreducible rings

4 (1971) $31-34$

I.M.S. Dey

Free products and residual nilpotency

1 (1969) $11-13$

Phil Diamond

Fractional iteration of functions of two variables (A)

2 (1970) 138-139

Submartingales and stochastic stability

10 (1974) $271-276$

J. B. Diaz and John G. Leschen

A remark on a mean value theorem of Alexander Weinstein in Generalized Axially Symmetric Potential Theory

J. B. Diaz and R. Výborný

On some mean value theorems of the differential calculus

S. E. Dickson and G. M. Kelly

Interlacing methods and large indecomposables

Vlastimil Dlab

Structure of perfect rings

Ian Wilson Doherty

Almost periodic generalized functions (A)

$\begin{array}{ccc}9 & (1973) & 1-9 \\ 5 & (1971) & 227-238 \\ 3 & (1970) & 337-348 \\ 2 & (1970) & 117-124 \\ 10 & (1974) & 311-312\end{array}$

John Donaldson

Asymptotic estimates of the errors in the numerical integration of analytic functions (A)

1 (1969) 431

W. Dorfler

On the edge-reconstruction of graphs

$10 \quad(1974) \quad 79-84$

Margaret Anne Dow

Maximum principles for some quasilinear elliptic-parabolic operators (A)

P. van den Driessche and R. D. Braddock

Asymptotic expansion of integrals occurring in linear wave theory

$9 \quad(1973) \quad 157-159$

7 (1972) $121-130$

J. E. Drummond

An existence theorem for differential equations

A formula for accelerating the convergence of a general series

J. L. Dyer

A five lemma for free products of groups with amalgamations 
Robert John Dyne

An investigation of the collision broadening of spectral lines (A)

R. E. Edwards

Inequalities related to those of Hausdorff-Young

R. E. Edwards and Kenneth A. Ross

Helgason's number and lacunarity constants

S. Elhay

Optimal quadrature

G. C. Elton

See A. L. Andrew

Ian Graham Enting

An analysis of critical phenomena (A)

$10 \quad(1974) \quad 471-472$

M. W. Evans

Some topics in non-singular rings (A)

I. H. Farouqi

On an infinite int egral linear group (A)

On an infinite integral linear group

\section{R. J. Faudree}

Regular metabelian groups of prime-power order

V. Ficker

On the equivalence of a countable disjoint class of sets of positive measure and a weaker condition than total $\sigma$-finiteness of measures

Alessandro Figa-Talamanca and J. F. Price

Rudin-Shapiro sequences on compact groups

P. D. Finch

Sasaki projections on orthocomplemented posets

On the lattice structure of quantum logic

Quantum logic as an implication algebra

Orthogonality relations and orthomodularity

On the structure of Stone lattices

On the Mobius function of a non-singular binary relation

Peter D. Finch

Macroscopic descriptions of microscopic phenomena

Queueing and the provision of service facilities

Peter D. Finch and Elizabeth Heathcote

Characters for summary functions associated with cartesian products

D. G. Fitz-Gerald and G. B. Preston

Divisibility of binary relations

W. E. Fitzgibbon

Nonlinear differential equations in reflexive Banach spaces

Isidore Fleischer

A final remark on extending to strict total orders in modules

Edward Formanek

The zero divisor question for supersolvable groups

Colin D. Fox

On a problem in the theory of ordered groups

J. M. Foyster and C. B. G. McIntosh

The classification of some spherically symmetric space-time metrics

Jorgen Segerlund Frederiksen

The analytic properties of the triangle and box diagram amplitudes (A)

Linda J. Freede

See Robert C. Thompson 


\section{Peter Freyd}

Aspects of topoi

Aspects of topoi: Corrigenda and acknowledgements

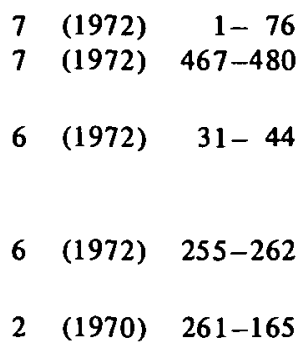

M. Frick and M. F. Newman

Soluble linear groups

Terence M. Gagen and Mark P. Hale, Jr

On the maximal normal prime-nilpotent subgroup of a prime-solvable group

$2(1970) \quad 261-165$

Spaces having no large dyadic subspace

J. L. B. Gamien

See R. J. Gaudet

J. M. Gandhi

Some group laws equivalent to the commutative law

$2(1970) \quad 335-345$

B. J. Gardner

A note on types

Rings whose modules form few torsion classes

(1970) 275-276

$4 \quad(1971) \quad 355-359$

B. P. Garfoot

Perturbation methods applied to the nonlinear symmetric bending of circular elastic plates (A)

$5 \quad(1971) \quad 427-428$

Herbert S. Gaskill

On the relation of a distributive lattice to its lattice of ideals

On the relation of a distributive lattice to its lat tice of ideals: Corrigendum

R. W. Gatterdam

The computability of group constructions II

R. J. Gaudet and J. L. B. Gamlen

An elementary proof of part of a classical conjecture

David B. Gauld

Tubular neighbourhoods for submersions of topological manifolds

Anthony V. Geramita, Norman J. Pullman, and Jennifer S. Wallis

Families of weighing matrices

7 (1972) $377-385$

8 (1973) $\quad 317-318$

A. R. Gifkins

An algebraic approach to Runge-Kutta methods (A)

J. R. Giles

Smooth semi-inner-product spaces (A)

A Banach space with support homeomorphism is reflexive

8 (1973) $\quad 27-60$

3 (1970) 289-292

8 (1973) 93-102

10 (1974) 119-122

9 (1973) $145-146$

2 (1970) 142-143

$5 \quad(1971) \quad 187-189$

Robert Ian Goldblatt

Metamathematics of modal logic (A)

$10 \quad(1974) \quad 479-480$

G. H. Golub and E. Seneta

Computation of the stationary distribution of an infinite Markov matrix

Computation of the stationary distribution of an infinite stochastic matrix of special form

8 (1973) $333-341$

$10 \quad(1974) \quad 255-261$

Colin C. Graham

The weak density of the non-invertible elements of a commutative Banach algebra

Douglas D. Grant, D. A. Holton, and K. L. McAvaney

Sabidussi-ty pe theorems for stability

$\begin{array}{ccc}4 & (1971) & 179-182 \\ 10 & (1974) & 95-105 \\ 9 & (1973) & 33-42 \\ 2 & (1970) & 89-93\end{array}$

J. A. Graves and J. J. Malone

Embedding near domains

J. D. Gray

The weak closure of the set of singular elements in a Banach algebra 
D. G. Green

Extensions of a semilattice by an inverse semigroup

\section{Fletcher Gross}

Elementary abelian operator groups

Finite groups which are the product of two nilpotent subgroups

Marvin W. Grossman

Uniqueness of invariant means on certain introverted spaces

\section{J. R. J. Groves}

On varieties of soluble groups

Varieties of soluble groups and a dichotomy of P. Hall

Varieties of soluble groups (A)

On varieties of soluble groups II

A. K. Gupta

See U. B. Tewari

Narain Gupta and Frank Levin

Some symmetric varieties of groups

Narain D. Gupta, Horace Y. Mochizuki, and Kenneth W. Weston

On groups of exponent four with generators of order two

J. A. Guthrie

Ascoli theorems and the pseudocharacter of mapping spaces

J. A. Guthrie, D. F. Reynolds, and H. E. Stone

Connected expansions of topologies

9 (1973) $21-31$

7 (1972) $91-100$

9 (1973) 267-274

9 (1973) 109-120

5 (1971) 95-109

$5 \quad(1971) \quad 391-410$

6 (1972) $159-160$

7 (1972) $437-441$

Anthony John Guttmann

Numerical studies in phase transitions (A)

William Haebich

The multiplicator of a regular product of groups

The multiplicator of various products of groups (A)

\section{Mark Hale Jr}

See Terence M. Gagen

T. E. Hall

On regular semigroups whose idempotents form a subsemigroup

On the lattice of congruences on a regular semigroup

On regular semigroups whose idempotents form a subsemigroup: Addenda

Christopher John Harman

A discrete analytic theory for geometric difference functions (A)

\section{J. Harman}

A note on a discrete analytic function

A new definition of discrete analytic functions

L. F. Harris

An index of $P$. Hall for varieties of groups

Varieties and section closed classes of groups (A)

$\begin{array}{ccc}3 & (1970) & 97-105 \\ 10 & (1974) & 135-142 \\ 10 & (1974) & 403-408 \\ 9 & (1973) & 259-265 \\ & & \\ 2 & (1970) & 427-428 \\ 7 & (1972) & 279-296 \\ 7 & (1972) & 461-462\end{array}$

B. Hartley and D. McDougall

Injective modules and soluble groups satisfying the minimal condition for normal subgroups

4 (1971) 113-135

B. Hartley

See also R. M. Bryant

George Havas, G. E. Wall, and J. W. Wamsley

The two generator restricted Burnside group of exponent five

$10 \quad(1974) \quad 459-470$

C. R. Heathcote and J. W. Pitman

An inequality for characteristic functions

$\begin{array}{ll}(1969) & 195-208 \\ (1969) & 231-235 \\ (1970) & 287-288 \\ & \\ (1973) & 299-300 \\ & \\ (1974) & 123-134 \\ (1974) & 281-291 \\ & \\ (1972) & 399-405 \\ (1973) & 475-476\end{array}$

Elizabeth Heathcote

6 (1972) $1-9$

See Peter D. Finch 
Joachim A. Hempel

Su perlinear variational boundary value problems and nonuniqueness $(\mathbf{A})$

J. A. Hempel, G. R. Morris and N. S. Trudinger

On the sharpness of a limiting case of the Sobolev imbedding theorem

C. C. Heyde and J. R. Lestie

Improved classical limit analogues for Galton-Watson processes with or without immigration

\section{J. L. Hickman}

Some definitions of finiteness

Some definitions of finiteness: Corrigenda

On a cardinal equation in set theory

\section{John Lewellyn Hickman}

An investigation of several finiteness criteria relative to Zermelo-Fraenkel set theory (A)

John C. Higgins

Representing $N$-semigroups

\section{Denis Higgs}

Boolean-valued equivalence relations and complete extensions of complete boolean algebras

\section{James Murray Hil}

Some partial solutions of finite elasticity (A)

\section{A. Holton}

Completely semi-stable trees

See also Douglas D. Grant

\section{J. R. Holub}

Bounded approximate identities and tensor products

\section{S. Hoo}

See E. Chislett

\section{Joe Howard and Kenneth Melendez}

Sufficient conditions for a continuous linear operator to be weakly compact

\section{J. M. Howie and B. M. Schein}

Anti-uniform semilattices

\section{T. Howroyd}

Cancellative medial groupoids and arithmetic means

O. F. Hughes

A useful relationship in the conformal mapping of quadrilaterals

David C. Hunt

Character tables of certain finite simple groups

R. P. Hunter and L. W. Anderson

A remark on compact semigroups having certain decomposition spaces embeddable in the plane

A remark on compact semigroups having certain decomposition spaces embeddable in the plane: Corrigendum

D. G. Hurley and J. Imberger

Surface and internal waves in a liquid of variable depth

D. G. Hurley

See also P. F. Siew

J. Imberger

See D. G. Hurley $\begin{array}{lll}5 & (1971) & 421-422 \\ 3 & (1970) & 369-373\end{array}$

5 (1971) 145-155

$5 \quad(1971) \quad 321-330$

$6 \quad(1972) \quad 319$

6 (1972) 447-457

7 (1972) 300

1 (1969) 115-125

3 (1970) $65-72$

7 (1972) 463-465

9 (1973) 355-362

7 (1972) 443-445

7 (1972) 183-190

1 (1969) 263-268

8 (1973) $17-21$

9 (1973) 99-104

$5 \quad(1971) \quad 1-42$

4 (1971) 137-139

4 (1971) 432

1 (1969) 29-46 
Index

Volume 1 (1969) - Volume 10 (1974)

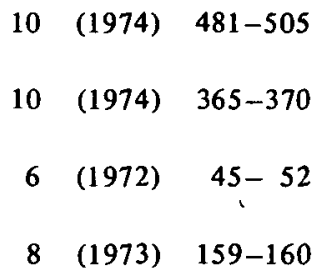

Kiyoshi Iséki

On common fixed points of mappings

G. Ivanov

Non-local rings whose ideals are all quasi-injective

George Ivanov

On matrix methods in ring theory (A)

Masako lzumi and Shin-ichi lzumi

Absolute Nörlund summability of Fourier series of functions of bounded variation

Absolute Riesz summability of Fourier series and their conjugate series

Integrability of trigonometric series III

Divergence of Fourier series

Divergence of Fourier series: Corrigenda

Uniform convergence and everywhere convergence of Fourier series. I

Uniform convergence and every where convergence of Fourier series. II

Divergence of Fourier series: Corrigenda. II

Shin-ichi Izumi

See Masako Izumi

Pushpa Jain

See M. K. Singal

P. Jambor

See L. Bican

Ian Robert James

Concepts of independence for bounded-sum variables (A)

9 (1973) $\quad 469-470$

Ludvik Janos

An application of combinatorial techniques to a topological problem

$9 \quad(1973) \quad 439-443$

T. L. Jenkins

See L. C. A. van Leeuwen

L. S. Jennings

Orthogonal transformations and improperly posed problems (A)

S. Jeyamma

The interrelations among various spaces of distributions

J. A. Johnson

A remark on a theorem of Caradus

$\begin{array}{ll}(1970) & 111-123 \\ (1970) & 217-229 \\ (1971) & 205-219 \\ (1973) & 289-304 \\ (1973) & 319-320 \\ (1973) & 321-335 \\ (1973) & 337-342 \\ (1974) & 152-160\end{array}$

Lee W. Johnson

Unicity in the uniform approximation of vector-valued functions

N. L. Johnson

A characterization of generalized Hall planes

A note on generalised Hall planes

$$
\begin{array}{lll}
9 & (1973) & 303-304 \\
4 & (1971) & 263-271 \\
6 & (1972) & 355-356 \\
3 & (1970) & 193-198 \\
6 & (1972) & 61-67 \\
8 & (1973) & 151-153 \\
6 & (1972) & 83-94 \\
6 & (1972) & 95-105 \\
7 & (1972) & 105-111 \\
7 & (1972) & 391-405
\end{array}
$$

A. S. Jones

Laminar forced convection at low Péclet number

Laminar forced convection at low Péclet number. II

Michael J. Kallaher

A note on planes of characteristic three

L.-C. Kappe and W. P. Kappe

On three-Engel groups

W. P. Kappe

See L.C. Kappe

A. G. Kartsatos

See N. A. Tserpes 
G. M. Kelly

See S. E. Dickson

T. Kepka

See L. Bican

Bruce W. King

Presentations of metacyclic groups

$\begin{array}{ccc}8 & (1973) & 103-131 \\ 10 & (1974) & 317-318 \\ & & \\ 4 & (1971) & 205-209 \\ 6 & (1972) & 407-415 \\ & & \\ 1 & (1969) & 127-136 \\ 1 & (1969) & 169-173 \\ 7 & (1972) & 367-376\end{array}$

Bruce Whitfield King

Normal structure of $p$-groups (A)

P. B. Kirkpatrick

Generalization of Hall planes of odd order

A characterization of the Hall planes of odd order

Martin Kleiber and W. J. Pervin

Semifield metric spaces

A generalized Banach-Mazur theorem

C. J. Knight

Remainders of metric completions

John Howard Knight

Solutions of the nonlinear diffusion equation: existence, uniqueness, and estimation (A)

9 (1973) $477-478$

J. Knopfmacher

On Lie algebra obstructions

$1 \quad(1969) \quad 281-288$

Ian Walker Knowles

The limit-point and limit-circle classification of the Sturm-Liouville operator $(p y)^{\prime}+q y(\mathbf{A})$

10 (1974) $315-316$

L. G. Kovács and M. R. Vaughan-Lee

A problem of Hanna Neumann on closed sets of group words

$5 \quad(1971) \quad 341-342$

L. G. Kovács

See also Warren Brisley

See also M. S. Brooks

See also R. M. Bryant

D. B. Lahiri

Some arithmetical identities for Ramanujan's and divisor functions

R. Lalithambal

On theorems of Thornton

Alan Lambert

Unitary equivalence and reducibility of invertibly weighted shifts

Panayotis Th. Lambrinos

A note on quasi-u niform continuity

Quasi proximal continuity

Klaus Lamotke

See Philip T. Church

H. Lausch

An application of a theorem of Gaschutz

H. Lausch and A. Makan

On a relation between the Fitting length of a soluble group and the number of conjugacy classes of its maximal nilpotent subgroups

1 (1969) 307-314

$6 \quad$ (1972) 211-212

$5 \quad$ (1971) $157-173$

8 (1973) 389-392

9 (1973) $89-98$

Meira Lavie

Analytic iterations on Riemann surfaces

L. C. A. van Leeuwen and T. L. Jenkins

A supernilpotent non-special radical class

John C. Lennox and James Wiegold

Some remarks on coherent soluble groups

$\begin{array}{ccc}1 & (1969) & 381-384 \\ 1 & (1969) & 3-10 \\ 1 & (1969) & 183-194 \\ 9 & (1973) & 343-348 \\ 10 & (1974) & 277-279\end{array}$


Philip A. Leonard

Adders for the patterned starter in some non-abelian groups

10 (1974) $321-323$

Yu Kiang Leong

The CREAM conjecture and certain abelian-by-nilpotent varieties (A)

$7 \quad(1972) \quad 459-460$

Y. K. Leong

The CREAM conjecture for the subvarieties of certain abelian-by-nilpotent varieties

$10 \quad(1974) \quad 429-451$

John G. Leschen

See J. B. Diaz

J. R. Leslie

See C. C. Heyde

Richard A. Levaro

A note on flabby sheaves

$7 \quad(1972) \quad 387-389$

Frank Levin

One variable equations over semigroups

2 (1970) 247-252

See also Narain Gupta

\section{Steve Ligh}

On boolean near-rings

On the commutativity of near-rings III

A generalization of a theorem of Wedderburn

John W. Lloyd

Inductive and projective limits of smooth topological vector spaces

John Lloyd

Two topics in the differential calculus on topological linear spaces (A)

W. S. Lo

Spectral approximation theorems for bounded linear operators

1 (1969) 375-379

$6 \quad(1972) \quad 459-464$

8 (1973) $181-185$

$6 \quad(1972) \quad 227-240$

9 (1973) $305-306$

$8 \quad(1973) \quad 279-287$

\section{F. Peter Lockett}

See Alan R. Camina

\section{J. A. Loustau}

On a class of power-associative periodic rings

$5 \quad(1971) \quad 357-362$

E. R. Love

Functions of asymptotic expansions

$6 \quad(1972) \quad 307-312$

David Lowell Lovelady

A necessary and sufficient condition for exponentially bounded existence and uniqueness

\section{R. J. Loy}

Continuity of derivations on topological algebras of power series

Identities in tensor products of Banach algebras

Uniqueness of the Fréchet space topology on certain topological algebras

Local a nalytic structure in certain commutative topological algebras

Richard J. Loy

Commutative Banach algebras with non-unique complete norm topology

P. E. Lush and J. W. Stephenson

The practical use of the Rayleigh-Ritz method in compressible flow

I. D. Macdonald

Computer results on Burnside groups

1 (1969) $419-424$

2 (1970) 253-260

4 (1971) $1-7$

6 (1972) $161-167$

10 (1974) $409-420$

$4 \quad(1971) \quad 85-95$

$9 \quad(1973) \quad 433-438$

Sheila Oates Macdonald

Locally finite varieties of groups arising from Cross varieties

Locally finite varieties of groups arising from Cross varieties: Corrigendum

Laws in finite strictly simple loops

See also John Cossey

4 (1971) 211-215

4 (1971) 431-432

9 (1973) 349-354 
Sheila Oates Macdonald and Anne Penfold Street

On Conway's conjecture for integer sets

$8 \quad(1973) \quad 355-358$

J. M. Mack

A note on simultaneous approximation

On the continued fraction algorithm

Simultaneous diophantine approximation (A)

(1970) $81-83$

$\begin{array}{lll}3 & (1970) & 413-422\end{array}$

$6 \quad(1972) \quad 317-318$

\section{W. R. Madych}

See G. D. Allen

Kenneth D. Magill, Jr

Zero-free fortifying homomorphisms and semigroups of relations

1 (1969) 213-230

Kurt Mahler

An arithmetic remark on entire periodic functions

An elementary existence theorem for entire functions

Arithmetical properties of the digits of the multiples of an irrational number

On a class of diophantine inequalities

On the coefficients of transformation polynomials for the modular function

On rational approximations of the exponential function at rational points

\section{J. J. Mahony}

Three-dimensional Stokes flow

5 (1971) 191-195

5 (1971) $415-419$

$8 \quad(1973) \quad 191-203$

$8 \quad(1973) \quad 247-259$

$10 \quad$ (1974) $197-218$

10 (1974) 325-335

A. R. Makan

On some aspects of finite soluble groups (A)

The Fitting length of a finite soluble group and the number of conjugacy classes of its maximal nilpotent subgroups

On saturated formations whose projectors are complemented

7 (1972) $77-89$

$6 \quad(1972) \quad 157-158$

6 (1972) 213-226

$\begin{array}{lll}6 & (1973) & 239-248\end{array}$

\section{A. Makan}

See H. Lausch

Wilfred Gordon Malcolm

Ultraproducts and higher order models (A)

8 (1973) $315-316$

\section{J. J. Malone}

See J. A. Graves

See B. C. McQuarrie

George M. Marinoff

Magneto-viscous effects on resistive plasma instabilities (A)

10 (1974) 143-144

Jorge Martinez

The hyper-archimedean kernel sequence of a lattice-ordered group

$10 \quad(1974) \quad 337-349$

R. F. Matlak

An autonomous system of differential equations in the plane

Study of limit cycles of an autonomous system of differential equations (A)

1 (1969) 391-395

$7 \quad(1972) \quad 309-311$

K. L. McAvaney

See Douglas D. Grant

P. J. McCarthy

Matchings in graphs

9 (1973) 141-143

D. J. McCaughan

Subnormal structure in infinite soluble groups (A)

Subnormal structure in some classes of infinite groups

D. J. McCaughan and D. McDougall

The subnormal structure of metanilpotent groups

D. McDougall

See B. Hartley

See D. J. McCaughan

$\begin{array}{llll}7 & (1972) & 457-458\end{array}$

8 (1973) 137-150

6 (1972) 287-306 


\section{R. McGough}

A note on a theorem of Sah

$3 \quad(1970) \quad 269-271$

C. B. G. McIntosh

Cosmological models with $n$ fluids (A)

$4 \quad(1971) \quad 141-142$

See also J. M. Foyster

Stephen K. McKee

Orthogonal expansions and transforms of vector-valued measures and functions (A)

9 (1973) $151-152$

Geoffrey J. McLachlan

The errors of allocation and their estimators in the two-population discrimination problem (A)

James M. McPherson

A family of non-invertible prime links

Wild knots and arcs in 3-space (A)

A family of non-invertible prime links: Corrigendum

B. C. McQuarrie and J. J. Malone

Endomorphism rings of non-abelian groups

J. C. Meakin

Congruences on orthodox semigroups (A)

S. M. A. Meggitt

The stability of massive hydrogen stars near the main sequence (A)

Kenneth Melendez

See Joe Howard

T. Memp

Interior preserving maps

Stephen Meskin

Some varieties without the amalgam embedding property

Peter D. Meyer

An orthomodular poset which does not admit a normed orthovaluation

Gordon V. Miles

Wave propagation in weakly nonlinear dispersive systems (A)

9 (1973) $149-150$

4 (1971) $105-108$

4 (1971) 283-284

5 (1971) $141-143$

$3 \quad(1970) \quad 349-352$

$2(1970) \quad 285-286$

$4 \quad(1971) \quad 287$

J. B. Miller

A characterization of weak projectability

1 (1969) 209-212

$1 \quad(1969) \quad 417-418$

$3 \quad(1970) \quad 163-170$

$2(1970) \quad 429-431$

$8 \quad(1973) \quad 205-209$

J. Susan Milton and Chris P. Tsokos

On a random solution of a nonlinear perturbed stochastic integral equation of the Volterra type

9 (1973) 227-237

Donald P. Minassian

Remark on ordered abelian groups

$5 \quad(1971) \quad 411-413$

P. E. H. Minchin

Quantum mechanics and many-body problems. Effective operators in atomic energy level $Z$-expansion calculations (A)

8 (1973) $155-156$

H. Y. Mochizuki

See S. Bachmuth

Horace Y. Mochizuki

See Narain D. Gupta

Bertram Mond

On the complex complementarity problem

9 (1973) 249-257

B. Mond

See B. D. Craven

G. P. Monro

Small sets with large power sets

$8 \quad(1973) \quad 413-421$ 
R. A. R. Monzo

On categorical semigroups

T. Bryant Moodie

On the propagation, reflection, and transmission of transient cylindrical shear waves in nonhomogeneous four-parameter viscoelastic media

\section{H. G. Moore}

Subdirectly irreducible rings - some pathology

On commutativity in certain rings

Free algebra structure: categorical algebras

G. R. Morris

See J. A. Hempel

Sidney A. Morris

Varieties of topological groups

Varieties of topological groups II

Varieties of topological groups III

Varieties of topological groups (A)

Free products of topological groups

A universal semigroup

Sidney A. Morris and H. B. Thompson

Topologies on finite groups

Invariant metrics on free topological groups

\section{A. Mukherjea}

A Stone-Weierstrass theorem for random functions

See also N. A. Tserpes

F. J. Narcowich

See G. D. Allen

L. Narici, G. Bachman and E. Beckenstein

The weak density of the non-invertible elements of a commutative algebra

P. Nermec

See L. Bican

B. H. Neumann

Editorial

M. F. Newman

Just non-finitely-based varieties of groups

See also M. S. Brooks

See also M. Frick

D. F. Nicholls

Inference problems for vector linear time series models (A)

Rodney Nillsen

Extreme point properties of fixed-point sets

Rodney Victor Nillsen

Fixed points of semigroups in a Choquet simplex and compactification (A)

E. S. Noussair

Differential equations in Banach spaces

B. J. Noye

On a class of differential equations which model tide-well systems

On the differential equation for the conventional tide-well system

The response of lake levels to an unsteady wind stress

G. C. O'Brien

Almost periodic and quasi-periodic solutions of differential equations (A)

Kevin C. O'Meara

Right orders in full inear groups (A)
9 (1973) 61- 68

8 (1973) $397-411$

1 (1969) 353-355

2 (1970) 107-115

3 (1970) 207-215
5 (1971) 387-390

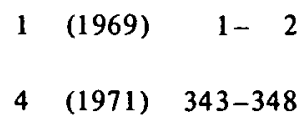

$7 \quad(1972) \quad 451-452$

6 (1972) 241-249

6 (1972) $315-316$

9 (1973) 219-226

3 (1970) 391-411

7 (1972) 251-267

8 (1973) 423-433

7 (1972) 453-454

6 (1972) $468-470$ 
D. L. Outcalt and Adil Yaqub

A commutativity theorem for rings

A commutativity theorem for power-associative rings

W. J. Padgett

Mean-square stability of a class of stochastic integral equations

A. G. Pakes

An asymptotic result for a subcritical branching process with immigration

K. J. Palmer

Averaging and integral manifolds (II)

See also W. A. Coppel

David L. Parrott

On the Mathieu groups $M_{22}$ and $M_{11}$ (A)

Glynn Washington Peady

The generation and propagation of waves in an ocean of variable depth (A)

K. R. Pearson

On the units of a modular group ring

On the units of a modular group ring II

William M. Pender

Classical groups over division rings of characteristic two

Classical groups over division rings of characteristic two: Corrigenda and an acknowledgement

Elizabeth Pennington

Trifactorisable groups

Paul Pentony

Laws in torsion-free nilpotent varieties with particular reference to the laws of free nilpotent groups (A)

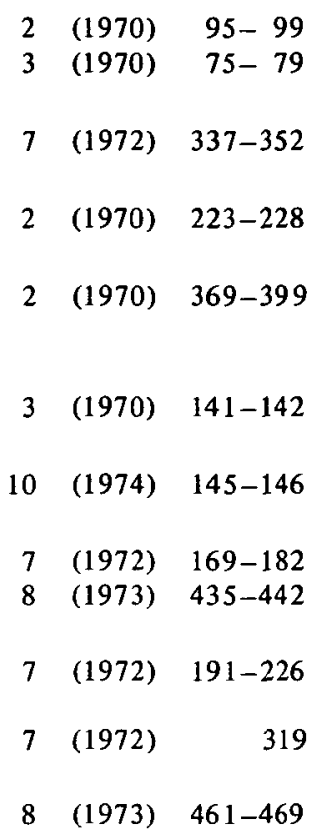

$5 \quad(1971) \quad 283-284$

\section{R. Perry}

See A. R. Amir-Moéz

\section{W. J. Pervin}

See Howard Anton

See Martin Kleiber

H. F. Petersons

Disturbance daily variation (A)

$3 \quad(1970) \quad 140$

B. B. Phadke

Conditions for a plane projective metric to be a norm

A. F. Pillow

Diffusion and convection in viscous flow

E. J. G. Pitman

Simple proofs of Steck's determinantal expressions for probabilities in the Kolmogorov and Smirnov tests

7 (1972) 227-232

J. W. Pitman

See C. R. Heathcote

John Poland

Extensions of finite nilpotent groups

$2(1970) \quad 267-274$

\section{A. J. van der Poorten}

Generalisations of Turán's main theorems on lower bounds for sums of powers

A generalisation of Turan's main theorems to binomials and logarithms

Perfect approximation of functions

9 (1973) $49-54$

2 (1970) $145-164$

Jack R. Porter

Minimal first countable spaces

2 (1970) 15- 37

2 (1970) 183-195

5 (1971) 117-126

3 (1970) $55-64$ 
Neil Charles Powers

Operator theory on quaternionic Hilbert spaces (A)

7 (1972) 297-299

G. B. Preston

See D. G. Fitz-Gerald

J. F. Price

$\left(L^{p}, L^{q}\right)$-multiplier problems (A)

4 (1971) 281-282

See also Alessandro Figa'-Talamanca

Stephen J. Pride

Residual properties of free groups II

Residual properties of free groups (A)

7 (1972) 113-120

Norman J. Pullman

See Brian R. Alspach

See Anthony V. Geramita

M. P. Quine

Multitype Galton-Watson processes (A)

John Randolph

A solvability condition for finite groups with nilpotent maximal subgroups

$10 \quad(1974) \quad 477-478$

K. M. Rangaswamy and N. Vanaja

A note on modules over regular rings

7 (1972) $301-302$

3 (1970) $273-276$

Stuart Rankin

See Daihachiro Sato

K. P. S. Bhaskara Rao and M. Bhaskara Rao

A note on the countable chain condition and sigma-finiteness of measures

$6 \quad(1972) \quad 349-353$

M. Bhaskara Rao

See K. P. S. Bhaskara Rao

M. Rama Mohana Rao and Chris P. Tsokos

Integro-differential equations of Volterra type

V. Venu Gopal Rao

Convergence criteria for Fourier series

4 (1971) 57-62

See also Badri N. Sahney

V. Venu Gopal Rao and B. N. Sahney

On a sequence of Fourier coefficients

$5 \quad(1971) \quad 343-349$

M. A. Rashid

See S. Asghar

H. Rasmussen

Steady viscous axisymmetric flow associated with rotating disks (A)

Ivan L. Reilly

A generalized contraction principle

$3(1970) \quad 9-22$

4 (1971) 259-262

N. R. Reilly

Free generators in free inverse semigroups

Free generators in free inverse semigroups: Corrigenda

D. F. Reynolds

See J. A. Guthrie

A. H. Rhemtulla and Anne Penfold Street

Maximal sum-free sets in finite abelian groups

2 (1970) 289-297

P. F. Rhodes-Robinson

Fundamental singularities in the theory of water waves with surface tension

Fundamental singularities in the theory of water waves with surface tension: Corrigenda

$\begin{array}{rrr}2 & (1970) & 144 \\ 10 & (1974) & 359-363 \\ 7 & (1972) & 407-424 \\ 9 & (1973) & 479-480\end{array}$

P. Ribenboim

On the extension of orders in ordered modules

2 (1970) $317-333$

$3(1970) \quad 432$

$2(1970) \quad 81-88$ 
On the extension of orders in ordered modules: Corrigenda

J. A. Rickard

A two parameter eigenvalue problem

R. D. Riess

Hermite-Fejér interpolation at the 'practical' Chebyshev nodes

David A. Robbins

Existence of a bounded approximate identity in a tensor product

A constructive proof of Gelbaum's theorem on tensor products

A. M. Roberts

Moving loads on elastic and thermoelastic solids (A)

William Ian Robertson

Wave packet scattering and time delay (A)

J. Robinson

Mixtures of distributions (A)

Peter M. Robinson

The estimation of continuous-time systems using discrete data (A)

\section{Keith William Robson}

Strongly magnetic, rapidly rotating main-sequence stars (A)

Robert Edward Robson

Transport phenomena in neutral and ionized gases (A)

Vijay K. Rohatgi

On probabilities of large deviations

\section{Dieter K. Ross}

The Dirichlet boundary value problem for two non-overlapping spheres

Kenneth A. Ross

See R. E. Edwards

Don Row

A homomorphism theorem for projective planes

Homomo rphisms of sharply transitive projective planes

Allen Maurice Russell

Functions of bounded $k^{\text {th }}$ variation and Stieltjes type integrals (A)

Edgar A. Rutter, Jr

Four-fold torsion theories

D. M. Ryan

Transformation methods in nonlinear programming (A)

Gabriel Sabbagh

On properties of countable character

Badri N. Sahney and V. Venu Gopal Rao

Error bounds in the approximation of functions

Error bounds in the approximation of functions: Corrigenda and an acknowledgement

$\begin{array}{rrr}4 & (1971) & 155-158 \\ 4 & (1971) & 361-366 \\ 10 & (1974) & 475-476 \\ 10 & (1974) & 1-8 \\ 6 & (1972) & 478-479 \\ 4 & (1971) & 183-192 \\ 6 & (1972) & 11-18 \\ 6 & (1972) & 480\end{array}$

B. N. Sahney

See also V. Venu Gopal Rao

Ken W. Sarkies

Studies in surface physics (A)

9 (1973) $309-310$

Daihachiro Sato and Stuart Rankin

Entire functions mapping countable dense subsets of the reals onto each other monotonically

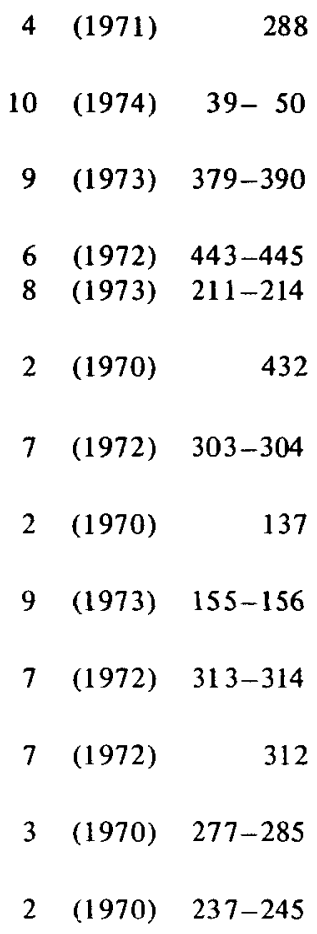

2 (1970) 


\section{J. L. Schiff}

Relations between boundaries of a riemannian manifold

$$
\begin{array}{lll}
6 & (1972) & 25-30 \\
8 & (1973) & 443-459
\end{array}
$$

\section{Schroder}

Solid convergence spaces

John W. Schutz

Foundations of special relativity: kinematic axioms for Minkowski space-time (A)

$6 \quad(1972) \quad 465-467$

S. D. Scott

Near-rings and near-ring modules (A)

4 (1971) 143-144

E. Seneta

On invariant measures for simple branching processes (Summary)

2 (1970) 359-362

See also G. H. Golub

A. G. Shannon and Leon Bernstein

The Jacobi-Perron algorithm and the algebra of recursive sequences

David Shield

Nilpotency and related properties of group extensions

$8 \quad(1973) \quad 261-277$

P. F. Siew and D. G. Hurley

Diff raction of planetary waves by a semi-infinite plate

Howard L. Silcock

Soluble groups satisfying the minimal condition for normal subgroups (A)

\section{Brailey Sims}

A characterization of Banach-star-algebras by numerical range

On numerical range and its application to Banach algebras (A)

M. K. Singal and Shashi Prabha Arya

A note on order paracompactness

\section{K. Singal and Pushpa Jain}

On subparacompact and countably subparacompact spaces

\section{Bhagat Singh and R. S. Dahiya}

Nonoscillation of third order retarded equations

\section{Narendra Kumar Sinha}

Propagation of magnetohydrodynamic shock waves in stellar interiors (A)

K. G. Smith

High Reynolds number flow between torsionally oscillating disks

P. W. Smith

See G. D. Allen

Raymond E. Smithson

Topologies generated by relations

1 (1969) 297-306

Robert L. Snider

Complemented hereditary radicals

$4 \quad(1971) \quad 307-320$

T. Soundararajan and K. Venkatachaliengar

On the Krull Galois theory for non-algebraic extension fields

4 (1971) 145-154

$6 \quad(1972) \quad 145-156$

9 (1973) 307-308

4 (1971) 193-200

7 (1972) 307-308

4 (1971) 273-279

$5 \quad(1971) \quad 289-304$

$10 \quad(1974) \quad 9-14$

$2 \quad(1970) \quad 287-288$

2 (1970) $55-79$

\section{Bruce Southcott}

Two-variable laws for a class of finite simple groups

\section{T. P. Speed}

Profinite posets

$4 \quad(1971) \quad 367-387$

$10 \quad(1974) \quad 85-89$

D. Spoerel

A remark on Ribenboim's paper, 'On the extension of orders in ordered modules' 
Joseph Mendel Steiner

Thermal convection in a non-newtonian fluid (A)

9 (1973) $153-154$

J. W. Stephenson

See P. E. Lush

Ian N. Stewart

Finiteness conditions in soluble groups and Lie algebras

9 (1973) $43-48$

P. K. Stewart

Inelastic collision processes in the Townsend-Huxley diffusion experiment

(A)

$8 \quad(1973) \quad 479-480$

A. N. Stokes

Differential inequalities and the matrix Riccati equation (A)

A special property of the matrix Riccati equation

9 (1973) $315-317$

$10 \quad(1974) \quad 245-253$

H. E. Stone

See J. A. Guthrie

T. Storer

Hurwitz on Hadamard designs

\section{A. R. Stralka}

The topological structure of $D$-classes

G. E. Strecker

Epireflection operators vs perfect morphisms and closed classes of epimorphisms

\section{Anne Penfold Street}

Maximal sum-free sets in cyclic groups of prime-power order

Maximal sum-free sets in abelian groups of order divisible by three

Maximal sum-free sets in abelian groups of order divisible by three: Corrigendum

See also Sheila Oates Macdonald

See also A. H. Rhemtulla

Charles Swartz

The product of vector-valued measures

S. Świerczkowski

Homogeneous Lie algebras

W. N.-C. Sy

Microturbulent magnetoactive plasmas and polarized radiation from the solar corona (A)

$\begin{array}{lll}4 & (1971) & 109-112 \\ 1 & (1969) & 289-295 \\ 7 & (1972) & 259-366 \\ 4 & (1971) & 407-418 \\ 6 & (1972) & 439-441 \\ 7 & (1972) & 317-318\end{array}$

G. Szekeres

Polyhedral decompositions of cubic graphs

D. G. Tacon

The conjugate of a smooth Banach space

Compactness-type problems in topological vector spaces. A treatment mainly from the viewpoint of non-standard analysis (A)

Peter James Taylor

The effect of beam seas on a stationary ship in shallow water (A)

U. B. Tewari

Isomotphisms of some convolution algebras and their multiplier algebras

U. B. Tewari and A. K. Gupta

The algebra of functions with Fourier transforms in a given function space

S. Therianos

See Robert C. Thompson

Harold Bevan Thompson

Some existence problems in differential equations approached through functional analysis (A)

$\begin{array}{lrr}8 & (1973) & 359-366 \\ 4 & (1971) & 349-353 \\ 9 & (1973) & 312 \\ 8 & (1973) & 367-387 \\ 2 & (1970) & 415-425 \\ 5 & (1971) & 429-430 \\ 7 & (1972) & 447 \\ 7 & (1972) & 321-335 \\ 9 & (1973) & 73-82\end{array}$

9 (1973) $147-148$ 
H. B. Thompson

See Sidney A. Morris

Robert C. Thompson and Linda J. Freede

Eigenvalues of partitioned hermitian matrices

$3 \quad(1970) \quad 23-37$

Robert C. Thompson and S. Therianos

Inequalities connecting the eigenvalues of a hermitian matrix with the eigenvalues of complementary principal submatrices

$6 \quad$ (1972) $117-132$

P. J. Thomson

Phase measurements and narrow spectral band inference (A)

M. C. Thornton

Torsion topological groups with minimal open sets

$\mathrm{K}$. Tronson

The limits of time stepping when used to solve the long wave equations numerically

8 (1973) $321-331$

Neil S. Trudinger

On the analyticity of generalized minimal surfaces

$5 \quad(1971) \quad 315-320$

N. S. Trudinger

See J. A. Hempel

N. A. Tserpes and A. G. Kartsatos

On the equivalence of invariant integrals and minimal ideals in semigroups

1 (1969) 269-278

N. A. Tserpes and A. Mukherjea

Some problems on idempotent measures on semigroups

2 (1970) 299-315

Chris P. Tsokos

See J. Susan Milton

See M. Rama Mohana Rao

N. Vanaja

See K. M. Rangaswamy

J. C. Varley

Relative annihilators in semilattices

9 (1973) $169-185$

M. R. Vaughan-Lee

Generating groups of nilpotent varieties

On product varieties of groups

3 (1970) 145-154

5 (1971) 239-240

See also L G. Kovács

K. Venkatachaliengar

See T. Soundararajan

Mark Villarino

See Ronald Alter

Cornelis Paul Vlagsma

On the $S$-semigroups of automata (A)

10 (1974) 473-474

A. R. Vobach

A theorem on homeomorphism groups and products of spaces

1 (1969) 137-141

Two structure theorems for homeomorphism groups

4 (1971) 63- 68

R. Výborný

See J. B. Diaz

Graeme C. Wake

Equations of heat conduction with slow combustion (A)

1 (1969)

144

G. E. Wall

See George Havas

Jennifer Wallis

Hadamard designs

Integer matrices obeying generalized incidence equations 
A skew-Hadamard matrix of order 92

Combinatorial matrices (A)

5 (1971) 203-204

See also Joan Cooper

Jennifer S. Wallis

See Anthony V. Geramita

Jennifer Wallis and Albert Leon Whiteman

Some classes of Hadamard matrices with constant diagonal

$7 \quad(1972) \quad 233-249$

W. D. Wallis

Certain graphs arising from Hadamard matrices

On the relationship between graphs and partially balanced incomplete block designs

Construction of strongly regular graphs using affine designs

Construction of strongly regular graphs using affine designs: Corrigenda

On a problem of $\mathrm{K}$. A. Bush concerning Hadamard matrices

See also S. B. Conlon

J. M. Wamsley

The deficiency of finite groups (A)

The multiplicator of finite nilpotent groups

See also George Havas

5 (1971) 285-286

\section{J. N. Ward}

On finite groups admitting automorphisms with nilpotent fixed-point groups

On finite soluble groups and the fixed-point groups of automorphisms

On groups admitting a noncyclic abelian automorphism group

Nilpotent signalizer functors on finite groups

Martin Ward

Totally unordered subsets of a partially well ordered set

Eric Warren

A note on a result of $\mathrm{K}$. D. Magill, Jr

A. M. Watts

A singular perturbation problem with a turning point

The temperature outside a heated plume

Richard Weiss

Numerical procedures for Volterra integral equations (A)

B. R. Wenner

Sums of finite-dimensional spaces

1 (1969) $325-331$

1 (1969) $425-430$

4 (1971) $41-49$

$\begin{array}{llll}5 & (1971) & 431\end{array}$

$6 \quad(1972) \quad 321-326$

Kenneth W. Weston

See Narain D. Gupta

Albert Leon Whiteman

See Jennifer Wallis

Anthony K. Whitford

Characterization of vector-valued Laplace transforms and moment sequences (A)

$1 \quad(1969) \quad 143$

3 (1970) $1-8$

Wilbur Whitten

On prime noninvertible links

James Wiegold

Schunk classes are nilpotent product closed

Ext $(Q, Z)$ is the additive group of real numbers

5 (1971) 281-282

5 (1971) $375-378$

9 (1973) 363-366

9 (1973) 367-377

2 (1970) 179-182

7 (1972) 161-162

$5 \quad(1971) \quad 61-73$

5 (1971) 111-116

8 (1973) $\quad 477-478$

1 (1969) 357-361

See also John C. Lennox

H. Wiesler and G. Calugareanu jr

Remarks on triples in enriched categories

$8 \quad(1973) \quad 157-158$

5 (1971) $127-130$

1 (1969) $27-28$

1 (1969) 341-343

3 (1970) 375-383 


\section{F. W. Wilke}

Pseudo-fields and doubly transitive groups

$7 \quad(1972) \quad 163-168$

Neil H. Williams

Cardinal numbers with partition properties (A)

3 (1970) 143-144

N. H. Williams

An order property of partition cardinals

$\begin{array}{lll}3 & (1970) & 171-178\end{array}$

Transversals of squares

7 (1972) 353-358

A. G. Williamson

A note on the summation of an infinite series involving a hypergeometric function

10 (1972) 305-309

S. K. Wong

On a new finite non-abelian simple group of Janko

G. R. Wood

Semigroups of differentiable functions (A)

1 (1969) $59-79$

l. W. Wright

Some ordered topological groups (A)

9 (1973) $313-314$

$10 \quad(1974) \quad 313-314$

Sadayuki Yamamuro

On the semigroup of all continuous linear mappings on a Banach space

4 (1971) 201-203

\section{H. P. Yap}

Maximal sum-free sets in finite abelian groups

Maximal sum-free sets in finite abelian groups, II

Some remarks on stable graphs

4 (1971) 217-223

5 (1971) $43-54$

$10 \quad$ (1974) 351-357

Adil Yaqub

See D. L. Outcalt

Yeo Kok Chye

Minimal generating sets for some wreath products of groups

9 (1973) 127-136

Minimal number of generators of some classes of groups (A)

9 (1973) 301-302

David E. Zitarelli

Inverse subsemigroups of Rees matrix semigroups

9 (1973) 445-463 